\title{
Reliability and validity of neurobehavioral function on the Psychology Experimental Building Language test battery in young-adults
}

Brian J Piper, Shane T Mueller, Alexander R Geerken, Kyle L Dixon, Gregory Kroliczak, Reid HJ Olsen, Jeremy K Miller

Background. The Psychology Experiment Building Language (PEBL) software consists of over one-hundred computerized tests based on classic cognitive neuropsychology and behavioral neurology measures. Although the PEBL tests are becoming more widely utilized, there is currently very limited information about the psychometric properties of these measures. Methods. Study I examined inter-relationships among nine PEBL tests including indices of motor-function (Pursuit Rotor and Dexterity), attention (Test of Attentional Vigilance and Time-Wall), working memory (Digit Span Forward), and executive-function (PEBL Trail Making Test, Berg/Wisconsin Card Sorting Test, lowa Gambling Test, and Mental Rotation) in a normative sample ( $\mathrm{N}=189$, ages 18-22). Study II evaluated test-retest reliability with a two-week interest interval between administrations in a separate sample ( $N=79$, ages $18-22$ ). Results. Moderate intra-test, but low intertest, correlations were observed and ceiling/floor effects were uncommon. Sex differences were identified on the Pursuit Rotor (Cohen's $d=0.89$ ) and Mental Rotation ( $d=0.31$ ) tests. The correlation between the test and retest was high for tests of motor learning (Pursuit Rotor time on target $r=.86$ ) and attention (Test of Attentional Vigilance response time $r=.79$ ), intermediate for memory (digit span $r=.63$ ) but lower for the executive function indices (Wisconsin/Berg Card Sorting Test perseverative errors $=.45$, Tower of London moves $=.15$ ). Significant practice effects were identified on several indices of executive function. Conclusions. These results are broadly supportive of the reliability and validity of individual PEBL tests in this sample. These findings indicate that the freely downloadable, open-source, PEBL battery http://pebl.sourceforge.net is a versatile research tool to study individual differences in neurocognitive performance. 
1 Reliability and Validity of Neurobehavioral Function on the Psychology Experimental

2

3

\section{Building Language Test Battery in Young-Adults}

Brian J. Piperabc, Shane T. Muellerd, Alexander R. Geergen ${ }^{\mathrm{a}}$, Kyle L. Dixon ${ }^{\mathrm{ae}}$, Gregory Króliczak $^{\mathrm{f}}$, Reid H. J. Olsenc, \& Jeremy K. Miller ${ }^{\mathrm{a}}$

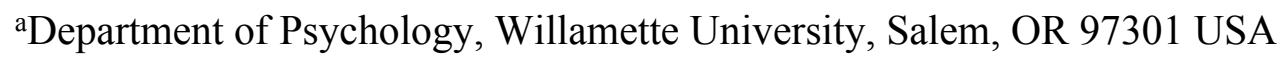

bDepartment of Psychology, Bowdoin College, Brunswick, ME 04011 USA

'Department of Behavioral Neuroscience, Oregon Health and Science University, Portland, OR 97239 USA

${ }^{\mathrm{d} D e p a r t m e n t}$ of Cognitive \& Learning Sciences, Michigan Technological University, Houghton, MI 49931 USA eDepartment of Psychology, University of New Mexico, Albuquerque, NM 87131 USA fAction and Cognition Laboratory, Department of Social Sciences, Institute of Psychology, Adam Mickiewicz University of Poznań, Poznań, Poland

Corresponding Author:

Brian J. Piper, Ph.D.

255 Main Street

Department of Psychology \& Neuroscience Program

Bowdoin College 
33 Brunswick, ME 04001

35

36

37

38

\section{4 psy391@gmail.com; bpiper@bowdoin.edu}

\section{Abstract}

Background. The Psychology Experiment Building Language (PEBL) software consists of over one-hundred computerized tests based on classic cognitive neuropsychology and behavioral neurology measures. Although the PEBL tests are becoming more widely utilized, there is currently very limited information about the psychometric properties of these measures. Methods. Study I examined inter-relationships among nine PEBL tests including indices of motor-function (Pursuit Rotor and Dexterity), attention (Test of Attentional Vigilance and TimeWall), working memory (Digit Span Forward), and executive-function (PEBL Trail Making Test, Berg/Wisconsin Card Sorting Test, Iowa Gambling Test, and Mental Rotation) in a normative sample ( $\mathrm{N}=189$, ages $18-22)$. Study II evaluated test-retest reliability with a twoweek interest interval between administrations in a separate sample $(\mathrm{N}=79$, ages 18-22).

Results. Moderate intra-test, but low inter-test, correlations were observed and ceiling/floor effects were uncommon. Sex differences were identified on the Pursuit Rotor (Cohen's $d=0.89$ ) and Mental Rotation $(d=0.31)$ tests. The correlation between the test and retest was high for tests of motor learning (Pursuit Rotor time on target $r=.86$ ) and attention (Test of Attentional Vigilance response time $r=.79$ ), intermediate for memory (digit $\operatorname{span} r=.63$ ) but lower for the executive function indices (Wisconsin/Berg Card Sorting Test perseverative errors $=.45$, Tower of London moves $=.15)$. Significant practice effects were identified on several indices of executive function.

Conclusions. These results are broadly supportive of the reliability and validity of individual PEBL tests in this sample. These findings indicate that the freely downloadable, open-source, 
58 PEBL battery http://pebl.sourceforge.net is a versatile research tool to study individual

59 differences in neurocognitive performance.

60 INTRODUCTION

61

62

63

64

65

66

67

A large collection of classic tests from the behavioral neurology and cognitive psychology fields have been computerized and made available (http://pebl.sf.net). This Psychology Experiment Building Language (PEBL) (Mueller, 2010, 2014a, 2014b; Mueller \& Piper, 2014) has been downloaded over 168 thousand times with $73 \%$ of downloads by institutions located outside of the United States, and used in scores of published manuscripts (e.g. Barrett \& Gonzalez-Lima, 2013; Danckert et al., 2012; Fox et al., 2013; Gonzalez-Giraldo et al. 2014, 2015a, 2015b; Piper, 2010; Piper et al. 2012; Premkumar et al., 2013; Wardle et al., 2012; Supplementary Table 1). The growth in PEBL use is likely due to three factors. First, PEBL is free while other similar programs (Robbins et al., 1994) have costs that preclude use by all but the largest laboratories and are beyond the capacities of the majority of investigators in developing countries. Second, PEBL is open-source software and therefore the computational operations are more transparent than may be found with proprietary measures. Third, the distributors of some commercial tests restrict test availability to those who have completed specific coursework whereas PEBL is available to anyone with an internet connection. This investigation reports on the use of nine PEBL measures including convergent and divergent validity (Study I) and test-retest reliability (Study II). These measures were selected because of their distinct neuroanatomical substrates (Figure 1) and because they measure theoretically important domains (Supplemental Table 2), are practical to administer, and are commonly employed in earlier biobehavioral investigations (Mueller \& Piper, 2014). A brief history of the more commonly utilized of these tests is provided below. 


\section{Digit Span}

82 The origins of digit span, an extremely simple test in which strings of numbers of

83 increasing length are presented and must be repeated back to the experimenter, are ambiguous

84 but procedures that are analogous to what are frequently employed today date back at least as far

85 as the pioneering developmental studies of Alfred Binet (Richardson, 2007). Although digit span

86 is frequently described as an index of working memory, the importance of attention for optimal

87 performance should not be underestimated (Lezak et al. 2012).

88 Rotary Pursuit

89 The rotary pursuit test measures motor performance by using a stylus to track a target that

90 moves clockwise at a fixed rate (Ammons, Alprin, \& Ammons, 1955). Procedural learning

91 deficits using the rotary pursuit have been shown among patients with Huntington's (Schmidtke

92 et al., 2002). As a result of the wide-spread use of the rotary pursuit in experimental psychology

93 laboratories, a computerized version was developed. Unfortunately, this version could only

94 generate linear target paths due to technical limitations at that time (Willingham, Hollier, \&

95 Joseph, 1995). The PEBL pursuit rotor is a more faithful version of the original rotary pursuit

96 distributed by Lafayette instruments. Importantly, prior computer experience does account for a

97 small portion of the variance in time on target (Piper, 2010).

98 Wisconsin Card Sorting Test

99 The Wisconsin Card Sorting Test is a classic neuropsychological measure of cognitive

100 flexibility and was originally developed at the University of Wisconsin following WWII by Esta

101 Berg and David Grant (Grant \& Berg, 1948). In the original version, participants sorted physical

102 cards into piles and determined the underlying classification principle by trial and error. Once

103 consistent correct matching was achieved, the principle would be changed. The subsequent 
104 development of a computerized version of this complex task made for both more efficient use of

105 the participants time and automated scoring (Lezak et al. 2012). Another key discovery was that

10664 cards could be used instead of 128 (Axelrod, Woodard, \& Henry, 1992; Fox et al. 2013).

107 Trail Making Test

108 The Trail Making Test is another of the oldest and most commonly employed

109 neurobehavioral measures (Lezak et al., 2012). The Trail Making Test is typically thought to

110 measure visual attention, mental flexibility, and executive functioning. The Trail Making Test

111 was contained in the Army General Classification Test, a precursor of the Armed Services

112 Vocational Aptitude Battery used by the United States military. The Trail Making Test involves

113 connecting dots arranged in a numbered sequence in ascending order (Part A) or numbers and

114 letters that alternate (Part B). Traditionally, performance on the Trail Making Test has been

115 timed with a stop-watch and the experimenter has to redirect the participant when they make an

116 error. Unlike the Halstead-Reitan Trail MakingTest (Gaudino, Geisler, \& Squires, 1995), the path

117 length is equal in Parts A and B of the PEBL Trail Making Test. Behavior on the Trail Making

118 Test is sensitive to wide variety of insults including alcoholism (Chanraud et al. 2009).

119 Mental Rotation Test

120 The mental rotation test has been an influential measure in cognitive psychology.

121 Participants must decide whether an image is rotated in space and there is a linear relationship

122 between the angle of rotation and decision time. Males exhibit better performance on spatial

123 ability tests with some evidence indicating that this robust sex difference (e.g. Yasen et al., 2015)

124 is detectable at very young ages (Linn \& Petersen, 1985; Moore \& Johnson, 2008).

125 Tower of London 
127 solution and move colored balls from their initial position to a new set of predetermined or goal

128 positions (Shallice, 1982). There are many variations on this "brain teaser" type task including

129 different levels of difficulty and construction (wood versus computerized) (Lezak et al. 2012).

130 An elevation in the number of moves to solve Tower of London type problems has been

131 documented among patients with brain damage and schizophrenia (Morris et al., 1995; Shallice, 132 1982).

133 Iowa Gambling Task

134 The Iowa Gambling Task was developed to model real world decision making in a

135 laboratory environment. Participants receive $\$ 2,000$ to start and must maximize their profit by

136 choosing cards from among four decks of which two typically result in a net gain (+\$250) and

137 two result in a net loss (-\$250). Although the Iowa Gambling Task has been employed with a

138 wide range of neuropsychiatric disorders, identification of a condition that consistently shows an

139 abnormality on this test has proved difficult with the possible exception of problem gamblers

140 (Buelow \& Suhr, 2009; Power, Goodyear, \& Crockford, 2012).

141 Test of Variables of Attention

142 The Test of Variables of Attention is an index of vigilance and impulsivity in which the

143 participant responds to a target but inhibits responses for non-target stimuli. Although continuous

144 performance tests were intended to discriminate children with, and without, Attention Deficit

145 Hyperactivity Disorder (Greenberg \& Waldman, 1993), the Test of Variables of Attention and

146 other similar instruments may have proved even more valuable in measuring attention as a

147 general construct and more specifically in evaluating the efficacy of cognitive enhancing drugs

148 (Huang et al. 2007). 
Another feature of the PEBL battery is that the key brain structures for these classic tasks

150 are reasonably well characterized based on both lesion studies and more recent neuroimaging

151 investigations (Figure 1). Importantly, as diffuse neural networks are responsible for complex

152 behaviors and the notion of a single neuroanatomical area underlying performance on a test risks

153 oversimplification, more comprehensive information can be found elsewhere (Demakis, 2004;

154 Gerton et al., 2004; Grafton et al., 1992; Hugdahl, Thomsen, \& Ersland, 2006; Jacobson et al.,

155 2011; Kaneko et al., 2011; Rogalsky, et al., 2012; Schall et al., 2013; Specht et al., 2009; Tana et

156 al., 2010; Zacks, 2008). Briefly, completing the rotary pursuit with the dominant (right) hand

157 results in a pronounced increase in blood flow in the left primary motor cortex, right cerebellum,

158 the supplementary motor area, and the left putamen (Grafton et al. 1992). Tasks that require

159 sustained attention engage the anterior cingulate and the insula (Tana et al., 2010). Digit Span

160 activates the left prefrontal cortex when examined with near-infrared spectroscopy (Kaneko et al.

161 2011). Whole brain comparison of Digit Span backward, relative for forward, using Position

162 Emission Tomography (PET) revealed blood flow elevations in the dorsal lateral prefrontal

163 cortex, left intraparietal lobule, and in Broca's area (Gerton et al., 2004). The Mental Rotation

164 Test results in a robust activation in the right intraparietal sulcus as well as in the frontal and

165 inferotemporal cortex (Jacobson et al. 2011). Executive function measures like the Trail Making

166 Test, Iowa Gambling Test, Tower of London, and Wisconsin Card Sorting Test have been

167 adapted from their clinical neuropsychological roots to be appropriate in a neuroimaging

168 environment. Part B of the Trail Making Test, relative to Part A, produces Blood Oxygen Level

169 Dependent elevations in the inferior middle frontal gyri (Jacobson et al. 2011). The left middle

170 frontal gyrus and right cerebellar tonsils show Tower of London difficulty dependent activations

171 as determined by both functional magnetic resonance imaging and PET. The left ventral medial 
172 prefrontal cortex is engaged during completion of the Iowa Gambling Task (Schall et al. 2013)

173 although lesion studies have produced conflicting evidence regarding the importance of this

174 structure (Shallice, 1982). The Wisconsin Card Sorting Test is a highly cognitively demanding

175 task which involves an extremely diffuse cortical network including the right middle frontal

176 gyrus as well as the left and right parietal lobule (Kaneko et al., 2011).

177 Previously, performance on three of the most prevalent executive function tests including

178 the Wisconsin (Berg) Card Sorting Test, Trail Making Test, and the Tower of London was

179 determined in a lifespan (age 5-87) sample. This investigation identified the anticipated "U-

180 shaped" association between age and performance on these PEBL tests (Piper et al. 2012). One

181 objective of the present report was to extend upon this foundation in a young-adult population by

182 further examining the utility of the three executive function indices as well as six other tests

183 including one (Dexterity) that is completely novel and another (Time-Wall) that is relatively

184 obscure. Each participant in Study I completed all nine measures so that score distributions and

185 the inter-test correlations could be evaluated. This information is necessary because PEBL

186 measures, particularly the indices of executive function (i.e. measures that involve the frontal

187 cortex, Lezak et al. 2012), are becoming increasingly utilized. The non-PEBL versions of several

188 tests (Tower of London, Mental Rotation Test, Trail Making Test, rotary pursuit, and digit span)

189 are often conducted using non-computerized methodology (Lezak et al., 2012) so it is currently

190 unclear whether prior data on convergent and discriminant validity will be applicable. Many

191 young adults have extensive experience with computerized measures so it is also crucial to

192 determine whether any measures have ceiling effects.

193 With the exception of a single pilot study (Piper, 2012), there is currently no information

194 about the test-retest reliability of individual PEBL tests or the battery. This dearth of data is 
195 unfortunate because the PEBL tests have already been employed in repeated measures designs

196 (Barrett \& Gonzalez-Lima, 2013; Premkumar et al., 2013; Wardle et al, 2012) and additional

197 information would aid in the interpretation of those findings. The consistency of measurement is

198 captured by two complementary measures. The correlation between the test and the retest

199 measures the relative consistency, and the effect size quantifies the absolute consistency in

200 performance.

201 There is a vast literature on the reliability of non-PEBL tests (Calamia, Markon, \&

202 Tranel, 2013; Lezak et al. 2012) and a few investigations with similar methodology or sample

203 characteristics similar to this report provide some context for the present endeavor. College-

204 students assessed on a computerized target tracking task showed a high correlation $(r=.75)$

205 across sessions separated by two weeks (Fillmore, 2003). Strong correlations $(r>.70)$ were also

206 noted on several indices of the Test of Variables of Attention among children completing that

207 vigilance measure with a nine-day inter-test interval (Learck, Wallace, \& Fitzgerald, 2004).

208 Veterans in their late-20s exhibited an intermediate $(r=.52)$ consistency across three sessions

209 (one/week) of a computerized Digit Span forward (Woods et al., 2010). The percentage

210 selections of the disadvantageous decks showed a moderate correlation $(r \geq .57)$ when the Iowa

211 Gambling Task was administered thrice on the same day (Lejuez et al., 2005) but limited

212 information is available at longer intervals (Buelow \& Suhr, 2009). The magnitude of practice

213 effects appears to be task dependent with slight changes identified for the Digit Span forward

214 (Woods et al. 2010) and the Test of Variables of Attention (Learck et al. 2004) but pronounced

215 improvements for the Iowa Gambling Task (Bechara, Damasio, \& Demasio, 2000; Lejuez et al.

216 2005). Executive function tasks that have a problem solving element may, once solved, have a

217 limited reliability (Lowe \& Rabbit, 1998). For example, the correlation of the first with the 
218 second 64-trials on the Berg Card Sorting Test was relatively low $(r=.31)$ (Fox et al., 2013). In

219 fact, the Wisconsin Card Sorting Test has been referred to as a "one shot test" (Lezak et al.

220 2012).

221 Two secondary objective of this report are also noteworthy. First, these datasets provided

222 an opportunity to identify any sex differences on the PEBL battery. As a general rule, males and

223 females are more similar than dissimilar on most neurocognitive measures. However, as noted

224 previously, the Mental Rotation Test provides a clear exception to this pattern (Linn \& Petersen,

225 1985; Moore \& Johnson, 2008). A robust male advantage was observed among children

226 completing the PEBL Pursuit Rotor task (Piper, 2010) and similar sex differences have been

227 identified with the non-computerized version(Willingham et al. 1995) of this test. However, sex

228 differences were most pronounced only at older (81+) but not younger (21-80) ages on a

229 computerized task with many similarities to the PEBL Pursuit Rotor (Stirling et al., 2013).

230 A final objective was to evaluate the different card sorting rules on the Berg Card Sorting

231 Task. The PEBL version of the Wisconsin Card Sorting Task has been employed in over a dozen

232 reports (e.g. Danckert et al., 2012; Fox et al., 2013; Piper et al., 2012; Wardle et al., 2011) and

233 may be the most popular of the PEBL tests. Importantly, the Berg Card Sorting Test was

234 programmed based on the definitions of perseverative responses and perseverative errors

235 contained in Esta Berg's 1948 report (Berg, 1948). Alternatively, the Wisconsin Card Sorting

236 Task distributed by Psychological Assessment Resources employs the subsequent definitions of

237 Robert Heaton and colleagues (Heaton et al., 1993).

238 Overall, this report provides data from two separate cohorts regarding the validity and

239 test-retest reliability of several PEBL measures. This psychometric information is fundamental

240 for others that may be considering using these tests. 
Participants

Participants (Study I: N = 189, 60.3\% Female, Age = 18.9 \pm 1.0 ; Study II: $\mathrm{N}=79,73.0 \%$

246 Female, Age $=19.1 \pm 0.1)$ were college students receiving course credit. The test sequence in

247 Study I was as follows: written informed consent, Tapping, Pursuit Rotor, Time-Wall, Trail-

248 Making Task, Digit-Span Forward, Berg Card Sorting Test, Mental Rotation, Iowa Gambling

249 Task, Tower-of-London, Dexterity, and the Test of Attentional Vigilance. Due to hardware

250 technical difficulties, data from the tapping motor speed test were unavailable. Half of these

251 measures (Time-Wall, Trail-Making Test, Digit-Span, Mental Rotation and the Test of

252 Attentional Vigilance) contain programming modifications relative to the PEBL battery 0.6

253 defaults and may be found at: https://github.com/stmueller/pebl-custom/tree/master/Piper-PeerJ-

$2542015 b$. All neurobehavioral assessments were completed on one of eight desktop computers

255 running Microsoft Windows. Each of these tests is described further below and screen shots

256 including instructions are in the Supplemental Figure 1. The number of tests was slightly reduced

257 to eight for Study II and the sequence was a written informed consent followed by Pursuit Rotor,

258 Trail-Making Test, Digit-Span, Test of Attentional Vigilance, Tower-of-London, Iowa Gambling

259 Task, and Time-Wall. As Study I identified subtle sex differences on Mental Rotation, this test

260 was not included in Study II. Similarly, Study I found that an abbreviated version of the TMT

261 could be a substitute for a longer version so a two-trial version was employed in Study II. Study

262 The interval between the test and retest was two weeks (mean $=14.4 \pm 0.2$ days, Min $=11$, Max

$263=24)$. This inter-test interval could be employed to examine the effects of a cognitively 
264 enhancing drug. All procedures are consistent with the Declaration of Helinski and were

265 approved by the Institutional Review Board of Willamette University.

266 PEBL Tests

267 Pursuit Rotor measures motor-learning and requires the participant to use the computer

268 mouse to follow a moving target on four-fifteen second trials. The target follows a circular path

269 (8 rotations per minute) and the time on target and error, the difference in pixels between the

270 cursor and target, were recorded (González-Giraldo et al., 2015; Piper, 2010).

271 Time-Wall is an attention and decision making task that involves assessing the time at

272 which a target, moving vertically at a constant rate, will have traveled a fixed distance. The

273 primary dependent measure is Inaccuracy, defined as the absolute value of the difference

274 between the participant response time and the correct time divided by the correct time (Minimum

$275=0.00$ ). The correct time ranged from 2.0 to 9.2 seconds with feedback ("Too short" or "Too

276 long") provided after each of the ten trials (Piper et al. 2012).

277

The Trail-Making Test is an index of executive function test and assesses set-shifting. In

278 Set A, the participant clicks on an ascending series of numbers (e.g. 1-2-3-4). In Set B, the

279 participant alternates between numbers and letters (e.g. $1-$ A $-2-$ B). The primary dependent

280 measure from the five trials is the ratio of total time to complete $\mathrm{B} / \mathrm{A}$ with lower values (closer to

281 1.0) indicative of better performance. Based on the findings of Study I with five trials, only the

282 first two trials were completed in Study II.

283 In the PEBL default Digit Span forward, strings of numbers of increasing length starting

284 with three were presented via headphones and displayed at a rate of one/second. Audio feedback

285 (e.g. "Correct" or "Incorrect") was provided after each of three trials at each level of difficulty.

286 The primary dependent measure was the number of trials completed correctly. 

cards into one of four piles based on a rule (color, shape, number) that changes. Feedback

289 (“correct!" or "incorrect”) was displayed for $500 \mathrm{~ms}$ after each trial. This test differs somewhat 290 from the version employed previously (Fox et al. 2013; Piper et al. 2011) in that the prior 291 selections were displayed (Supplementary Figure 1E). The primary dependent measure is the 292 percent of the 64 responses that were perseverative errors defined and coded according to the 293 Heaton criteria (Heaton et al., 1993) although the number of categories completed and 294 perseverative responses was also recorded.

In the PEBL Mental Rotation Test, the participant must decide whether two 2angle of rotation varied in $45^{\circ}$ increments $\left(-135^{\circ}\right.$ to $\left.+180^{\circ}\right)$. The percent correct and response time were the dependent measures.

In the PEBL Iowa Gambling Task, the four decks are labeled 1, 2, 3, and 4 rather than A, B, C, and D (Buelow \& Suhr, 2009). The primary dependent measure was the $\$$ at the end and response preference $[($ Deck $3+$ Deck 4$)-($ Deck $1+$ Deck 2) $]$ with Decks 3 and 4 being advantageous and Decks 1 and 2 being disadvantageous. The response to feedback and the

303 frequency different strategies were employed, e.g. payoff and then change piles (Win-Switch), 304 lose money but continue with the same pile (Lose-Stay), was also documented. In the Tower-of-London, the participant must form a plan in order to move colored disks, 306 one at a time, to match a specified arrangement. The number of points to solve twelve problems 307 (3 points/problem) and the average completion time/problem were recorded. Based on some 308 indications of ceiling effects in Study I, Study II employed a more challenging version of this 
309 task (Piper et al. 2012) with the primary measure being moves and completion time as a

310 secondary measure.

311 Dexterity is a recently developed test of fine motor function that consists of a circular

312 coordinate plane with the center of the circle (demarcated by a thin black line) at x,y positions

3130,0 . The goal is to move the cursor (depicted as a colored ball) to a target located at various

314 positions. Movement of the cursor is affected by a "noise" component complementing the

315 directional input from the analog mouse to create the effect of interference or "jittering" motion.

316 The effect is such that successful navigation of the coordinate plane using the mouse encounters

317 resistance to purposeful direction, requiring continual adjustment by the participant to maintain

318 the correct path to the target. Visual feedback is given by the use of a color system, wherein the

319 cursor shifts gradually from green to red as proximity to the target becomes lesser. The task

320 consists of 80 trials (10 per "noise" condition), ten seconds maximum in length, with preset noise

321 factors (ranging in intensity) and target locations standardized for consistency between

322 participants. A lack of input from the participant results in a gradual drift towards the center. At

323 the conclusion of each trial, the cursor location is reset to the origin. Completion time and Moves

324 were recorded with Moves defined as the change in the vector direction of the mouse while

325 course correcting toward the target (Supplemental Figure 1H).

326 Finally, in the Test of Attentional Vigilance, participants are presented with "go/no-go"

327 stimuli that they must either respond or inhibit their response. An abbreviated version (6 min)

328 was employed. The primary dependent measures were the reaction time and the variability of

329 reaction times. All PEBL source code from these studies is available at:

330 https://github.com/stmueller/pebl-custom ).

331 Data Analysis 
All analyses were conducted using Systat, version 13.0 with figures prepared using

333 Prism, version 6.03. Ceiling and floor effects were determined by examining score distributions

334 for any measure with $\geq 5 \%$ of respondents scoring at the maximum or minimum of the obtainable

335 range on that measure. As the PEBL default criteria for perseverative errors on the Berg Card

336 Sorting Test is currently very different than that employed by Heaton et al., 1993 in the

337 Wisconsin Card Sorting Test, secondary analyses were completed with each definition. Sex

338 differences in Study I and the magnitude of practice effects (Study II) were expressed in terms of

339 Cohen's $d$ (e.g. [Absolute value $\left(\right.$ Mean $_{\text {Retest }}-$ Mean $\left._{\text {Test }}\right) / S D_{\text {Test }}$ ] with $0.2,0.5$, and 0.8 interpreted

340 as small, medium, and large effect sizes. In Study II, correlation ( $\mathrm{r}$ and rho) and paired t-tests

341 were calculated on the test and retest values. Test-retest correlations $>0.7$ were interpreted as

342 acceptable (Nunnally, 1994) and $<0.3$ as unacceptable. The percent change was determined in

343 order to facilitate comparison across measures. 


\section{RESULTS}

346 Study I: Normative Behavior \& Inter-Test Associations

The nine PEBL tests may be organized into the following broad domains: motor function

348 (Pursuit Rotor and Dexterity), Attention (Test of Attentional Vigilance and Time-Wall),

349 Working-Memory (Digit Span), and Executive Functioning/Decision Making (Trail Making

350 Test, Tower of London, Berg Card Sorting Test, Iowa Gambling Test, and the Mental Rotation

351 Test). Table 1 shows that there were substantial individual differences in this sample. With the

352 exception of the Tower of London (Maximum Possible Points = 36), no test showed evidence of

353 ceiling or floor effects. The Berg criteria for coding perseverative responses resulted in a many

354 more than the Heaton criteria $\left(\operatorname{Mean}_{\text {Berg }}=30.8 \pm 6.9 \%\right.$, Mean $_{\text {Heaton }}=11.9 \pm 8.1 \%, t(172)=$

$35524.10, P<.0005)$. The difference for perseverative errors was more subtle but still significant

$356\left(\mathrm{Mean}_{\text {Berg }}=12.9 \pm 5.8 \%\right.$, Mean $\left._{\text {Heaton }}=11.0 \pm 6.4 \%, t(172)=3.79, P<.0005\right)$ on the Berg Card

357 Sorting Test.

358 Overall, sex differences were infrequent. On the Pursuit Rotor, the total time on target

359 was greater in males $(47.6 \pm 6.0)$ than females $(41.8 \pm 7.0 \mathrm{sec}, t(182)=5.79, P<.0005, d=$

360 0.89). Further analysis determined that target time in males was elevated by over 1,300 msec on

361 each trial relative to females (Figure 2A). On the Mental Rotation Test, there was no sex

362 difference in the number correct $($ Females $=72.8 \pm 17.9 \%$, Males $=74.9 \% \pm 19.4 \%, t(168)=$

363 0.47). Decision time was increased by the angle and the number correct decreased as the rotation

364 angle extended away from zero degrees in either direction (Figure 2B). The sex difference

$365 \quad($ Males $=2,377.8 \pm 795.8$, Females $=2,638.0 \pm 863.3)$ for overall response time was barely

366 significant $(t(168)=1.99, P<.05, d=0.31)$ with more pronounced group differences identified 
367 at specific angles (e.g. $-45^{\circ}, d=0.51$ ). Further, on the Iowa Gambling Test, total amount earned

368 at the end of the game did not show a sex difference $($ Males $=\$ 1,928.95 \pm 707.99$, Females $=$

$3691,858.02 \pm 755.47, t(180)=0.64, P=.52)$ but, following a loss, Males $(3.0 \pm 3.5)$ were $73.1 \%$

370 more likely on their following choice to select again from the same deck $($ Females $=1.7 \pm 2.8$,

$371 t(136.6)=2.86, P<.01, d=0.41)$.

372 Table 2 depicts the correlations among the tests. Generally, the association within

373 measures on a single test was moderate to high (e.g. Pursuit Rotor, Test of Attentional Vigilance,

374 Berg Card Sorting Test) whereas between tests Spearman rho values were typically lower. Lower

375 performance on the Pursuit Rotor (i.e. higher Error) was associated with less attentional

376 consistency (i.e. larger Test of Attentional Vigilance variability), longer times to complete

377 Dexterity, more Perseverative Errors on the Berg Card Sorting Test, greater Time-Wall

378 Inaccuracy, and lower Digit Span forward. There were also several correlations on the indices of

379 executive function. Individuals that performed less well on the Trail Making Test (i.e. higher B

380 to A ratios) scored lower on the Tower of London and the Berg Card Sorting Test. The

381 correlation between Berg Card Sorting Test perseverative errors when coded according to the

382 Heaton and default (Berg) criteria was moderately high. More correct Mental Rotation responses

383 also corresponded with higher performance on the Tower of London. Also noteworthy, the B to

384 A Ratio with all five trials showed a strong correspondence with only the first two Trail Making

385 Test trials $\left(r_{\mathrm{S}}(178)=+0.90, P<.0005\right.$, Figure $\left.2 \mathrm{C}\right)$.

386 Study II: Test-Retest Reliability

387 Figure 3 shows the test-retest correlations ranked from highest to lowest. Spearman and

388 Pearson correlations $\geq 0.7$ were interpreted as acceptable, $\geq 0.3$ and $<.7$ as intermediate, and

389 below 0.3 as unacceptable. Acceptable correlations were identified on the Pursuit Rotar and the 
390 Test of Attentional Vigilance. Digit Span, Time-Wall, and most measures on the Berg Card

391 Sorting Test were intermediate. Select correlations were below the acceptability cut-off for the

392 Iowa Gambling Task and the Tower of London. The reliability of secondary measures is also

393 listed on Supplemental Table 3. Most notably, reliability coefficients on the Berg Card Sorting

394 Test were equivalent for perseverative errors with the Berg and Heaton definitions.

395 Figure 4 depicts the absolute reliability in terms of effect size from the test to the retest

396 for the primary dependent measures with Supplemental Table 1 also containing secondary

397 indices. Consistent responding (i.e. no significant change) was observed for the number of moves

398 to solve the Tower of London. Slight, but significant $(P \leq .05)$ improvements were noted for

399 Digit Span forward and Response Time on the Test of Attentional Vigilance. Significant ( $\mathrm{p}<$

$400.01)$ practice effects with a small effect size $(d \geq .2)$ were identified for the variability of

401 responding on the Test of Attentional Vigilance, the response pattern on the Iowa Gambling

402 Tasks, the B to A ratio on the Trail Making Test as well as time to complete Part A, and

403 perseverative errors on the Berg Card Sorting task defined according to the Berg criteria.

404 Intermediate $(d \geq .6)$ practice effects were identified with increased time on target on the Pursuit

405 Rotor, decreased mean time to solve each Tower of London problem, faster completion of Part B 406 of the Trail Making Test, and heightened accuracy on Time-Wall.

407 Further analysis on the Iowa Gambling Tasks determined that he amount earned at the 408 end of each session did not appreciably change from the test $(\$ 1944.85 \pm 85.04)$ to the retest

$409(2,162.13 \pm 116.03, t(67)=1.59, P=.12 ; r(66)=.10, P=.40)$. However, the number of

410 selections from the disadvantageous decks ( 1 and 2$)$ decreased $10.3 \%$ from the test $(45.6 \pm 1.4)$

411 to the retest $(40.9 \pm 1.8, t(67)=2.66, P<.01, d=3.9 ; r(66)=.41, P<.0005)$. 


\section{DISCUSSION}

414 Study I: Normative Behavior \& Inter-Test Associations

415 The principle objective of the first study was to evaluate the utility of a collection of tests

416 from the PEBL battery including convergent and divergent validity. As also noted in the

417 introduction, there are some methodological differences between the PEBL and non-PEBL tasks.

418 The difference between using a stylus versus a computer mouse to track a moving target in the

419 Pursuit Rotar/Rotary Pursuit may not be trivial. The TOVA, but not the TOAV, includes

420 microswitches to record response time which may result in a higher accuracy than may occur

421 without this hardware. Finally, some of these instruments have a prolonged history (Lezak et al.

422 2012) and the dependent measures for some commercial tests (e.g. the WCST and perseverative

423 errors) have evolved over the past six decades (Berg, 1948; Grant \& Berg, 1948; Heaton et al.

424 1993) to be more complex than may be readily apparent based upon reading only the peer-

425 reviewed literature.

426 The nine measures in this dataset were chosen based on a combination of attributes

427 including assessing distinct neurophysiological substrates (Figure 1), theoretically meaningful

428 constructs (Supplemental Table 2), ease and speed of administration, and frequency of use in

429 earlier publications (Mueller \& Piper, 2014). Admittedly, a potential challenge that even

430 seasoned investigators have encountered with a young-adult "normal" population is that they can

431 quickly and efficiently solve novel problems which may result in ceiling effects (Yasen et al.

432 2015). However, a substantial degree of individual differences were identified on almost all

433 measures (Table 1). The only test where there might be some concern about score distribution

434 would be the points awarded on the Tower of London. A future study (e.g. testing the efficacy 
435 of a cognitive enhancing drug) might consider: 1) using alternative measures like completion

436 time; 2) choosing one of the ten other Tower of London already included, e.g. the test contained

437 in Piper et al., 2012, or, as the PEBL code is moderately well documented for those with at least

438 an intermediate level programming ability, to 3) develop their own more challenging test using

439 one of the existing measures as a foundation.

440 The inter-relationships among tests were characterized to provide additional information

441 regarding validity. For example, indices of attention showed some associations with both motor

442 function and more complex cognitive domains like memory. Overall, the relatively low

443 correlations $(\approx \pm 0.3)$ between the Trail Making Test, Tower of London, and Berg Card Sorting

444 Test, are congruent with the sub-component specificity of executive function domains (Miyake et

445 al., 2000; Smith et al. 2013). Similarly, the lack of association of the Iowa Gambling Task with

446 other executive function measures is generally concordant with prior findings (Buelow \& Suhr,

447 2009). These findings contribute to a much larger evidence base regarding the validity of

448 measures of the executive functions including volition, planning and decision making, purposive

449 action, and effective performance (Lezak et al. 2012; Smith et al. 2013).

450 This dataset also provided an opportunity to examine whether behavior on this battery

451 was sexually dimorphic. Previously, a small $(d=0.27)$ sex difference favoring boys (ages $9-13)$

452 was identified on the Pursuit Rotor (Piper, 2011). This same pattern was again observed but was

453 appreciably larger $(d=0.89)$ which raises the possibility that completion of puberty in this

454 young-adult sample may be responsible for augmenting this group difference. On the other hand,

455 in a prior study with 3-dimensional Mental Rotation images and a very similar sample (Yasen et

456 al., 2015), sex differences were noted but the effect size was larger $(d=0.54)$ than the present

457 findings $(d=0.31)$. As the PEBL battery currently uses simple 2-dimensional images, image 
458 complexity is likely a contributing factor. Sex differences were not obtained on Time-Wall,

459 Berg Card Sorting Tests, Trail-Making, or Tower of London tests which is in-line with earlier

460 findings (Piper et al. 2012).

461 The Berg Card Sorting Test may be the most frequently employed PEBL test in published

462 manuscripts. As both the Berg Card Sorting Test and the Wisconsin Card Sorting Test are based

463 on the same core procedures (Berg, 1948; Grant \& Berg, 1948), these tests appear quite similar

464 from the participant's perspective. However, the sorting rules of Heaton et al. (1993) are

465 considerably more complex than those originally developed (Berg, 1948; Grant \& Berg, 1948).

466 The finding that five of the correlations with other tests were significant and of the same

467 magnitude with both and Berg and Heaton rules provides some evidence in support of functional

468 equivalence of these tests.

469

Study II: Test-retest Reliability

470

The principle objective of the second study was to characterize the test-retest reliability

471 of the PEBL battery with a two-week interval. The correlation between the test and retest is

472 commonly obtained in these types of investigations (Calamia et al., 2013; Fillmore, 2003; Learck

473 et al., 2004; Lejuez et al., 2005; Lezak et al. 2012; Woods et al., 2010). It is also important to be

474 cognizant that the Pearson or the Spearman correlation coefficients may not fully describe the

475 consistency of measurement when the tested participants show an improvement but maintain

476 their relative position in the sample compared to each other. Therefore, a direct comparison

477 between the test and retest scores was also conducted to quantify the extent of any practice

478 effects.

479 The test-retest correlations were high $(\geq .70)$ for the Pursuit Rotor and Test of

480 Attentional Vigilance and moderate ( $\geq .30)$ for Digit Span, the Berg Card Sorting Test. Some 
481 measures on the Iowa Gambling Task and the Tower of London have test-retest reliabilities that

482 were low. It is noteworthy that there is no single value that is uniformly employed as the

483 minimum reliability correlation with some advocates of 0.7 or even 0.8 while others reject the

484 notion of an absolute cut-off(Calamia et al., 2013). In general, an extremely thorough meta-

485 analysis concluded that most tests employed by neuropsychologists have correlations above 0.7

486 with lower values observed for measures of memory and executive function (Calamia et al.,

487 2013). Many tests that are widely used clinically and for research have test-retest reliabilities

488 that are in the 0.3 to 0.7 range (Lowe \& Rabbitt, 1998). Direct comparison with other

489 psychometric reports is difficult because the test-retest intervals and the participant

490 characteristics were dissimilar but they are generally in line with expectations. For example, the

491 present findings $(r=.86)$ are slightly higher than what has been reported previously $(r=.75)$ for

492 a computerized Rotary Pursuit task (Fillmore, 2003). The Test of Variables Attention showed

493 very high correlations for response time variability $(r=.87)$ and response time $(r=.79)$ among

494 school-age children with a one-week interval (Leark et al. 2004) which is identical to the present

495 findings with the TOAV. Healthy (i.e. "normal") adults showed intermediate correlations on

496 both Part A $(r=.46)$ and Part B $(r=.44)$ of the paper version of the TMT with a 20 -week

497 interval (Matarazzo et al. 1974) which is lower than the present findings ( $r=.61$ to .74$)$. A

498 mixed (i.e. intact and brain damaged) sample showed moderately high consistency on the Digit

499 Span $(\mathrm{r}=.68)$ with an eleven month interval (Dikmen et al. 1999). Perhaps surprisingly, there is

500 currently very limited reliability data from the non-PEBL computerized versions of the Iowa

501 Gambling Task or the Wisconsin Card Sorting Test for comparative purposes. The pronounced

502 degree of improvement from the test to the retest, whether expressed as the percent change or in

503 terms of Cohen's $d$, are in accord with most earlier findings (Basso et al. 1999; Versavel et a. 
504 1997). Overall, it is important to emphasize that reliability is not an inherent characteristic of a

505 test but instead a value that is influenced by the sample characteristics and the amount of time

506 between the test and retest. The two-week interval would be applicable, for example, to assessing

507 the utility of a cognitive enhancing drug but longer intervals should also be examined in the 508 future.

509 Some procedural details of many of the PEBL tasks employed in this study are worthy of 510 consideration. The numbers presented in Digit Span and the cards in the Berg Card Sorting Test

511 are selected from a set of stimuli such that the retest will not be identical to the test. The degree

512 of improvement would likely be even larger without this feature. Although not the goal of this

513 report, we suspect that the magnitude of practice effects would be attenuated if alternative

514 versions of tests were employed for the test and the retest. This possibility is already pre-

515 programmed into the Trail Making Test and Tower of London. Similarly, the direction of

516 rotation could be set at clockwise for the test and counterclockwise for the retest if additional

517 study determined equivalent psychometric properties independent of the direction of target

518 rotation. Another strategy that could attenuate practice effects might be to increase the number of

519 trials, particularly on Time-Wall and the Trail Making Test, until asymptotic performance was

520 observed. Further discussion of the varied parameters and the evolution of the Iowa Gambling

521 Task is available elsewhere (Piper et al., in review).

522 General Discussion

523 The information obtained regarding the validity and reliability of the majority of PEBL

524 tests is broadly consistent with expectations (Lezak et al. 2012; Lowe et al., 1998) and indicates

525 that these tests warrant further use for basic and clinical research. The overall profile including

526 the distribution of scores, convergent and divergent validity, practice effects being of the 
527 anticipated magnitude, and, where applicable, internal consistency, as well as an expanding

528 evidence base (Mueller \& Piper, 2014), demonstrates that the Rotary Pursuit, Test of Attentional

529 Vigilance, Digit Span, and Trail Making Test are particularly appropriate for inclusion in

530 generalized batteries with participants that are similar to those included in this sample.

531 One task where the psychometric properties are concerning is the Iowa Gambling Task.

532 An improvement was noted in the response pattern from the test to the retest which is consistent

533 with what would be expected with this executive function test a priori. However, the correlation

534 between the test and retest was not even significant when the more conservative statistic

535 (Spearman rho) was examined. Perhaps, in order to attenuate the practice effect, two alternative

536 forms of the Iowa Gambling Task could be developed (e.g. version A where decks 3 and 4 are

537 advantageous and a version B where decks 3 and 4 are disadvantageous). In fact, even more

538 sophisticated alternative forms of the Iowa Gambling Task which vary based on task difficulty

539 are being developed by others (Xiao et al. 2013). Another modification which might benefit the

540 test-retest correlation would be to increase the salience of feedback that follows each trial. The

541 feedback was very salient in the original (i.e. non computerized) version of this task in that the

542 experimenter would give or take money after each trial (Bechara et al., 1994). Perhaps, the

543 psychometric properties of the PEBL Iowa Gambling Task would be improved if auditory

544 feedback was presented after each trial or there were a fixed interval between trials which would

545 encourage the participant to reflect on their previous selection. These procedural modifications

546 were made for a subsequent study (Piper et al. in review). Overall, additional study is warranted

547 to better appreciate the present findings as there is no long-term test-retest reliability with the

548 non-PEBL computerized Iowa Gambling Task (Buelow \& Suhr, 2009). However, given the 
549 limited evidence for convergent validity or test-retest reliability, prior findings with the PEBL

550 Iowa Gambling Task (Lipnicki et al., 2009); may need to be cautiously interpreted.

551 Three limitations of this report should also be acknowledged. First, the PEBL battery also

552 includes many other indices (e.g. Cori's block tapping test of visuospatial working memory, a

553 Continuous Performance Test of vigilance, a Stroop test of executive functioning). Only a subset

554 of the many PEBL tests were utilized due to time constraints (approximately one-hour of

555 availability for each participant). Future investigations may be designed to focus more narrowly

556 on specific domains (e.g. motor function). Second, a future objective would be to provide

557 further information regarding criterion validity, e.g. by determining the similarities, or

558 differences, between the Test of Variables of Attention and PEBL Test of Attentional Vigilance

559 in neurologically intact and various clinical groups as this information is mostly unavailable for

560 the PEBL tests (although see Danckert et al. 2012 which utilized the Berg Card Sorting Tests and

561 brain injured patients). Third, different versions of the Tower of London were used in Study I

562 and II which limits inferences across datasets for this measure. More broadly, the psychometric

563 information obtained is most pertinent to the specific tests employed with very specific test

564 parameters. The generalizability of these results to alternative versions of these measures (e.g. a

565 Tower of London with more trials), different sequences of tests, or different (shorter or longer)

566 test batteries will require verification. Fourth, the sample in both studies consisted of young-

567 adult college students, primarily Caucasian and from a middle-class background. There are those

568 that are quite articulate in outlining the limitations of this population (Henrich, Heine, \&

569 Norenzayan, 2010; Reynolds, 2010). The data contained in this report should just be viewed as

570 an important first step as further investigations with different ages, socioeconomic, and ethnic

571 groups is needed. 
573 greatly facilitated our understanding of individual differences in neurobehavioral function (Lezak

574 et al., 2012). We feel that the transparency of the PEBL battery extends upon this earlier work

575 and provides an important alternative to commercial tests. In addition, the ability of anyone with

576 a functional computer, independent of their academic degrees, to use PEBL contributes to the

577 democratization of science.

578 On the other hand, two considerations with PEBL and other similar open-source

579 applications should be acknowledged. First, the flexibility of PEBL also has clear drawbacks in

580 that each investigator can, in theory, modify a test's parameters to meet their own experimental

581 needs. If an investigator reports that they employed a particular test from a specific commercial

582 distributor, there is wide-spread agreement about what this means as many these tests often have

583 only limited modifiability. However, if an investigator changes a PEBL test but fails to make the

584 programming code available, then it is more difficult to critically evaluate research findings. The

585 second potential drawback with PEBL may be ethical. The prohibition against clinical

586 psychologists (American Psychological Association, 2002), but not others, making

587 neurobehavioral tests readily available is discussed elsewhere (Mueller \& Piper, 2014). The

588 accessibility of PEBL to anyone, including psychiatrists, neurologists, or cognitive

589 neuroscientists for research or teaching purposes is consistent with the ethos of science (Merton, 590 1979).

591 These findings also begin to aid comparisons with other older neurobehavioral test

592 batteries. Table 3 contrasts PEBL with the Behavioral Assessment and Research System (BARS)

593 and, perhaps the current "gold standard" of batteries, the Cambridge Neuropsychological Test

594 Automated Battery (CANTAB) in terms of intellectual origins, the not insignificant differences 
595 in price and transparency, and sample tests. The BARS system is based on the behavioral 596 analysis principles of B.F. Skinner and is designed for testing diverse populations including

597 those with limited education and prior computer experience (Rohlman et al. 2003). The

598 CANTAB battery was designed with an emphasis on translating preclinical findings to humans

599 (Robbins et al. 1994). Each of these platforms have their own advantages and disadvantages with

600 the strength of PEBL being the number of tests, limited cost, and modifiability.

601 CONCLUSION

602 In closing, this report provides key information regarding the test-retest reliability, 603 convergent and discriminant validity of many commonly employed PEBL tests. Our hope is that

604 thorough, but critical, investigations of the psychometric properties of this novel methodology in 605 normal (present study) and atypical populations will insure that PEBL will continue to be widely 606 used by investigators in basic and applied areas. This will foster further integration between

607 these fields and further advance our understanding of the genetic, biochemical, and

608 neuroanotomical substrates of individual differences in neurocognition.

609

610 ACKNOWLEDGEMENTS

611 The technical assistance of Christopher J. Fox, Vera E. Warren, Hannah Gandsey, Sari N.

612 Matisoff, and Donna M. Nolan is gratefully recognized. 
617 American Psychological Association. 2002. Ethical principles of psychologists and code of 618 conduct. American Psychologist 47: 1060-1073.

619

Ammons RB, Alprin SI, Ammons CH. 1955. Rotary pursuit performance as related to sex and age of pre-adult subjects. Journal of Experimental Psychology 49:127-133.

622

623 Axelrod BN, Woodard JL, Henry RR. 1992. Analysis of an abbreviated form of the Wisconsin 624 Card Sorting Test. Clinical Neuropsychology 6: 27-31.

625

626 Barrett DW, Gonzalez-Lima F. 2013. Transcranial infrared laser stimulation produces beneficial 627 cognitive and emotional effects in humans. Neuroscience 230: 13-23.

628

629 Bechara A, Damasio H, Damasio A. 2000. Emotion, decision making and the orbitofronal 630 cortex. Cerebral Cortex 10: 295-307.

631

632 Bechara A., Damasio AR, Damasio H, Anderson SW. 1994. Insensitivity to future consequences 633 following damage to human prefrontal cortex. Cognition 50: 7-15.

634

635 Basso M.R., Bornstein R.A., Lang J.M. 1999. Practice effects on commonly used measures of 636 executive function across twelve months. Clin Neuropsychol 13: 283-92. 
638 Berg EA. 1948. A simple objective technique for measuring flexibility in thinking. Journal of

639 General Psychology 39: 15-22.

640

641 Buelow MT, Suhr JA. 2009. Construct validity of the Iowa Gambling Task. Neuropsychology

642 Review 19: 102-114.

643

644 Calamia M, Markon K, \& Tranel D. The robust reliability of neuropsychological measures:

645 Meta-analyses of test-retest correlations. Clinical Neuropsychology 27: 1077-1105.

646

647 Carlin D, Bonerba J, Phipps M, Alexander G, Shapiro M, Grafman J. 2000. Planning

648 impairments in frontal lobe dementia and frontal lobe lesion patients. Neuropsychologia 38: 655649665.

650

651 Chanraud S, Reynaud M, Wessa M, Penttila J, Kostogiannia N, Cachia A, Artiges E, Delain F, 652 Perrin M, Aubin HJ, Cointepas Y, Martelli C, Martinot JL. 2009. Diffusion Tensor Tractography 653 in Mesencephalic bundles: Relation to mental flexibility in detoxified alcohol-dependent 654 subjects. Neuropsychopharmacology 34: 1223-1232.

655

656 Danckert J, Stöttinger E, Quehl N, Anderson B. 2012. Right hemisphere brain damage impairs 657 strategy updating. Cerebral Cortex 22: 2745-2760. 658 
659 Demakis GJ. 2004. Frontal lobe damage and tests of executive processing: A meta-analysis of

660 the category test, stroop test, and trail-making test. Journal of Clinical \& Experimental

661 Neuropsychology 26: 441-450.

662

663 Dikmen SS1, Heaton RK, Grant I, Temkin NR. 1999. Test-retest reliability and practice effects

664 of expanded Halstead-Reitan Neuropsychological Test Battery. Journal of the International

665 Neuropsychological Society 5: 346-356.

666

667

668 Fillmore MT. 2003. Reliability of a computerized assessment of psychomotor performance and 669 its sensitivity to alcohol-induced impairment. Perceptual Motor Skills 97: 21-34.

670

671 Fox CJ, Mueller ST, Gray ST, Raber J, Piper BJ. 2013. Evaluation of a short-form of the Berg

672 Card Sorting Test. PLoS One 8: e63885.

673

674 Gaudino EA, Geisler MW, Squires NK. 1995. Construct validity in the Trail Making Test: What 675 makes part B harder? Journal of Clinical \& Experimental Neuropsychology 17: 529-535.

676

677 Gerton BK, Brown TT, Meyer-Lindenberg A, Kohn P, Holt JL, Olsen RK, Berman KF. 2004.

678 Shared and distinct neurophysiological components of the digits forward and backward tasks as

679 revealed by functional neuroimaging. Neuropsychologia 42: 1781-1787.

680 
681 Grafton ST, Mazziotta JC, Presty S, Friston K.J, Frackowlak SJ, Phelps ME. 1992. Functional

682 anatomy of human procedural learning determined with regional cerebral blood flow and PET.

683 Journal of Neuroscience 12: 2542-2548.

684

685 Grant DA, Berg EA. 1948. A behavioral analysis of degree of reinforcement and ease of shifting

686 to new responses in Weigl-type card-sorting problem. Journal of Experimental Psychology 38:

$687 \quad 404-411$.

688

689 González-Giraldo Y, Rojas J, Novoa P, Mueller ST, Piper BJ, Adan A, Forero DA. 2014.

690 Functional polymorphisms in BDNF and COMT genes are associated with objective differences

691 in arithmetical functioning in a sample of young adults. Neuropsychobiology 70: 152-7. doi:

$692 \quad 10.1159 / 000366483$.

693

694 González-Giraldo Y, González-Reyes RE, Mueller ST, Piper BJ, Adan A, Forero DA. $2015 a$.

695 Differences in planning performance, a neurocognitive endophenotype, are associated with a

696 functional variant in PER3 gene. Chronobiology International 32: 591-595. doi:

$697 \quad 10.3109 / 07420528.2015 .1014096$.

698

699 González-Giraldo Y, Rojas J, Mueller ST, Piper BJ, Adan A, Forero DA. 2015b. BDNF

700 Val66Met is associated with performance in a computerized visual-motor tracking test in healthy

701 adults. Motor Control in press.

702 
703 Greenberg LM, Waldman ID. 1993. Developmental normative data on the Test of Variables of

704 Attention (T.O.V.A. $\left.{ }^{\text {TM}}\right)$. Journal of Child Psychology \& Psychiatry 34:1019-1030.

705

706 Heaton RK, Chelune GJ, Talley JL, Kay GG, Curtiss G. 1993. Wisconsin card sorting test

707 manual: Revised and expanded. Odessa: Psychological Assessment Resources.

708

709 Henrich J, Heine SJ, Norenzayan A. 2010. The weirdest people in the world. Behavioral \& Brain

710 Sciences 33: 61-83. doi: 10.1017/S0140525X0999152X.

711

712 Huang YS, Chao CC, Wu YY, Chen YY, Chen CK. 2007. Acute effects of methylphenidate on

713 performance during the Test of Variables of Attention in children with attention

714 deficit/hyperactivity disorder. Psychiatry \& Clinical Neurosciences 61: 219-225.

715

716 Hugdahl K, Thomsen T, Ersland L. 2006. Sex differences in visuo-spatial processing: An fMRI

717 study of mental rotation. Neuropsychologia 44: 1575-1583.

718

719 Jacobson SC, Blanchard M, Connolly CC, Cannon M, Garavan H. 2011. An fMRI investigation

720 of a novel analogue to the Trail-Making Test. Brain \& Cognition 77: 60-70.

721

722 Kaneko H, Yoshikawa T, Nomura K, Ito H, Yamauchi H, Ogura M, Honjo S. 2011.

723 Hemodynamic changes in the prefrontal cortex during digit span task: A near-infrared

724 spectroscopy study. Neuropsychobiology 63: 59-65.

725 
726 Lezak MD, Howieson DB, Bigler ED, Tranel D. Neuropsychological Assessment, 2012, fifth

727 edition, Oxford, New York.

728

729 Learck RA, Wallace DR, Fitzgerald R. 2004. Test-retest reliability and standard error of

730 measurement for the test of variables of attention (T.O.V.A.) with healthy school-age children.

731 Assesssment 11: 285-289.

732

733 Lejuez CW, Aklin WM, Richards JB, Strong DR, Karler CW, Read JP. 2005. The Balloon

734 Analogue Risk Task (BART) differentiates smokers and nonsmokers. Journal of Experimental 735 and Clinical Neuropsychology 11: 26-33.

736

737 Lezak MD, Howieson DB, Bigler ED, Tranel D. Neuropsychological Assessment ( $5^{\text {th }}$ ed), 738 Oxford: New York.

739

740 Linn M, Petersen A. 1985. Emergence and characterization of sex differences in spatial ability:

741 A meta-analysis. Child Development 56: 1479-1498.

742

743 Lipnicki DM, Gunga H, Belavy DL, Felsenberg D. Decision making after 50 days of simulated 744 weightlessness. Brain Research 1280: 84-89.

745

746 Lowe C., Rabbitt P. 1998. Test/re-test reliability of the CANTAB and ISPOCD

747 neuropsychological batteries: Theoretical and practical issues. Neuropsychologia 36: 915-923. 
749 Matarazzo JD, Wiens AN, Matarazzo RG, Goldstein SG. 1974. Psychometric and clinical test-

750 retest reliability of the Halstead impairment index in a sample of healthy, young, normal men.

751 Journal of Nervous and Mental Disease 158: 37-49.

752

753 Merton RK. 1979. The sociology of science: Theoretical and empirical investigations, University

754 of Chicago: Chicago.

755

756 Miyake A, Friedman NP, Emerson MJ, Witzki AH, Howerter A. 2000. The unity and diversity

757 of executive functions and their contributions to complex "Frontal Lobe" tasks: A latent variable 758 analysis. Cognitve Psychology 41: 49-100.

759

760 Moore DS, Johnson SP. 2008. Mental rotation in human infants: A sex difference. Psychological

761 Science 19: 1063-1066.

762

763 Morris RG, Rushe T, Woodruffe PWR, Murray RM. 1995. Problem solving in schizophrenia: A

764 specific deficit in planning ability. Schizophrenia Research 14: 235-246.

765

766 Mueller ST. 2010. A partial implementation of the BICA cognitive decathlon using the

767 Psychology Experiment Building Language (PEBL). International Journal of Machine

768 Consciousness 2: 2273-2288.

769

770 Mueller ST. 2014a. The Psychology Experiment Building Language, Version 0.14. Retrieved

771 from http://pebl.sourceforge.net. 
773 Mueller ST. 2014b. The PEBL Manual, Version 0.14, Lulu Press, ISBN 978-0557658176.

774

775 Mueller ST, Piper BJ. 2014. The Psychology Experiment Building Language (PEBL) and PEBL

776 test battery. Journal of Neuroscience Methods 222: 250-259. doi:

777 10.1016/j.jneumeth.2013.10.024.

778

779 Nunnally JC, Bernstein IH. Psychometric theory (3rd ed). New York: McGraw Hill, 1994.

780

781 Piper BJ. 2010. Age, handedness, and sex contribute to fine motor behavior in children. Journal 782 of Neuroscience Methods 195: 88-91. doi: 10.1016/j.jneumeth.2010.11.018.

783

784 Piper BJ. 2012. Evaluation of the test-retest reliability of the PEBL continuous performance test 785 in a normative sample. PEBL Technical Report Series [On-line], \#2012-05,

786 http://sites.google.com/site/pebltechnicalreports/home/2012/pebl-technical-report-2012-05

787

788 Piper BJ, Li V, Eowiz M, Kobel Y, Benice T, Chu A, Olsen RH, Rice DZ, Gray HM, Mueller 789 ST, Raber J. 2012. Executive function on the Psychology Experiment Building Language test 790 battery. Behavior Research Methods 44: 110-123.

791

792 Power Y, Goodyear B, Crockford D. 2012. Neural correlates of pathological gamblers preference

793 for immediate rewards during the Iowa Gambling Task: An fMRI study. Journal of Gambling 794 Studies 28: 623-636. 
796 Premkumar M, Sable T, Dhanwal D, Dewan, R. 2013. Circadian levels of serum melatonin and

797 cortisol in relation to changes in mood, sleep and neurocognitive performance, spanning a year

798 of residence in Antarctica. Neuroscience Journal 2013; 254090.

799

800 Reynolds CR. 2010. Measurement and assessment. Psychology Assessment 22: 1-4.

801

802 Richardson JTE. 2007. Measures of short-term memory: A historical review. Cortex 43: 635803650.

804

805 Robbins TW, James M, Owen AM, Sahakian BJ, McInnes L, Rabbitt P. 1994. Cambridge

806 Neuropsychological Test Automated Battery (CANTAB): A factor analytic study of a large

807 sample of normal elderly volunteers. Dementia 5: 266-281.

808

809 Rogalsky C, Vidal C, Li X, Damasio H. 2012. Risky decision-making in older adults without

810 cognitive deficits: An fMRI study of VMPFC using the Iowa Gambling Task. Social

811 Neuroscience 7: 178-190.

812

813 Rohlman DS, Gimenes LS, Eckerman DA, Kang SK, Farahat F, Anger WK. 2003. Development

814 of the Behavioral Assessment Research System (BARS) to detect and characterize neurotoxicity

815 in humans. Neurotoxicology 24: 523-531.

816 
817 Schall U, Johnson P, Lagopoulos J, Juptner M, Jentzen W, Thienel R, Dittmann-Balçar A,

818 Bender S, Ward PB. 2013. Functional brain maps of Tower of London performance: A positron

819 emission tomography and functional magnetic resonance imaging study. Neuroimage 20: 1154-

8201161.

821

822 Schmidtke K, Manner H, Kaufmann R, Schmolck H. 2002. Cognitive procedural learning in 823 patients with fronto-striatal lesions. Learning \& Memory 9: 419-429.

824

825 Shallice T. 1982. Specific impairments of planning. Philosophical Transactions of the Royal 826 Society of London B: Biological Sciences 298: 199-209.

827

828 Smith PJ, Need AC, Cirulli ET, Chiba-Falek O, Attix DK. 2013. A comparison of the Cambridge 829 Automated Neuropsychological Test Battery (CANTAB) with "traditional" neuropsychological 830 testing instruments. Journal of Clinical \& Experimental Neuropsychology 2013; 35: 319-328. 831 doi: 10.1080/13803395.2013.771618.

832

833 Specht K, Lie CH, Shah NJ, Fink GR. 2009. Disentangling the prefrontal network for rule 834 selection by means of a non-verbal variant of the Wisconsin Card Sorting Test. Human Brain 835 Mapping 30: 1734-1743.

836

837 Stirling LA, Lipsitz LA, Qureshi M, Kelty-Stephan DG, Goldberger AL, Costa MD. 2013. Use 838 of a tracing task to assess visuomotor performance: Effects of age, sex, and handedness. Journals 
839 of Gerontology. Series A: Biological Sciences \& Medical Sciences 68: 938-45. doi:

$840 \quad 10.1093 /$ gerona/glt003.

841

842 Tana MG, Montin E, Cerutti S, Bianchi AM. 2010. Exploring cortical attentional system by

843 using fMRI during a continuous performance test. Computational Intelligence \& Neuroscience

844 329213. doi: 10.1155/2010/329213.

845

846 Versavel M1, van Laack D, Evertz C, Unger S, Meier F, Kuhlmann J. 1997. Test-retest

847 reliability and influence of practice effects on performance in a multi-user computerized

848 psychometric test system for use in clinical pharmacological studies. Arzneimittelforschung

849 47(6):781-786.

850

851 Wardle MC, Hart AB, Palmer AA, Wit H. 2012. Does COMT genotype influence the effects of 852 d-amphetamine on executive functioning? Genes, Brain and Behavior 12: 13-20.

853

854 Willingham DB, Hollier J, Joseph J. 1995. A Macintosh analogue of the rotary pursuit task. 855 Behavior Research Methods 27: 491-495.

856

857 Woods DL, Kishiyama MM, Yund EW, Herron TJ, Edwards B, Poliva O, Hink RF, Reed B.

858 2010. Improving digit span assessment of short-term verbal memory. Journal of Clinical \&

859 Experimental Neuropsychology 33: 101-111. doi: 10.1080/13803395.2010.493149.

860 
861 Xiao L, Wood SMW, Denburg NL, Moreno GL, Hernandez M, Bechara A. 2013. Is there a

862 recovery of decision-making function after frontal lobe damage? A study using alternative

863 versions of the Iowa Gambling Task. Journal of Clinical \& Experimental Neuropsychology 35:

864 518-529. doi: 10.1080/13803395.2013.789484.

865

866 Yasen AL, Raber J, Miller JK, Piper BJ. 2015. Sex, but not Apolipoprotein E genotype, 867 contributes to spatial performance in young-adults. Archives of Sexual Behavior in press.

868

869 Zacks JM. 2008. Neuroimaging studies of mental rotation: A meta-analysis and review. Journal 870 of Cognitive Neuroscience 20: 1-19. 
872 Figure 1. Key brain areas as identified by neuroimaging and lesion studies and the

873 corresponding Psychology Experiment Building Language Tests.

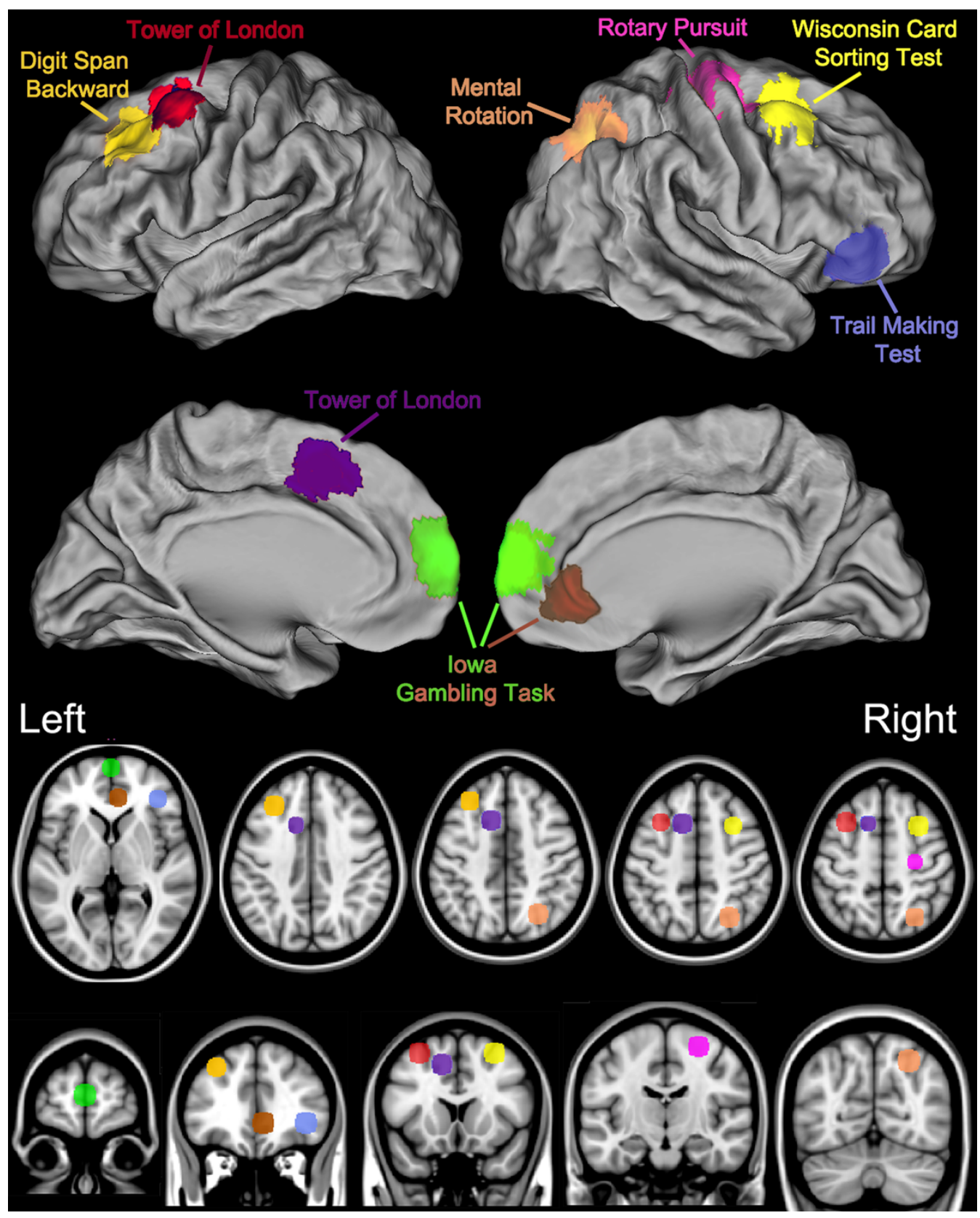


875 Figure 2. Neurobehavioral performance on Psychology Experiment Building Language (PEBL)

876 tests. A) Time on target on the Pursuit Rotor ( $* * * P<.0005$ versus Females); B) Decision time

877 and percent correct on the Mental Rotation $\left(* P<.0005\right.$ versus Angle $\left.=0^{\circ}\right)$; C) Scatterplot of

878 the ratio (Part B/Part A) of times to complete five versus two trials of the PEBL Trail-Making

879 Test.
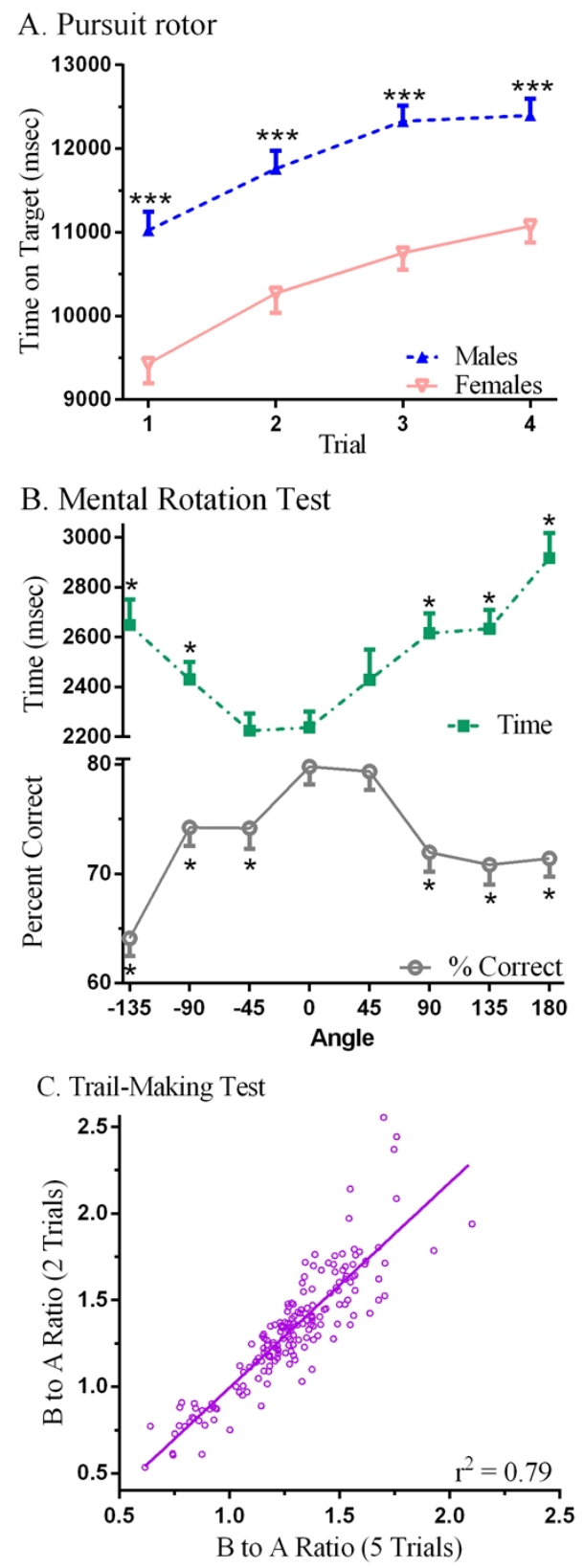
881

882 Figure 3. Test-retest correlations ranked from highest to lowest. For each Psychology

883 Experiment Building Language Test, the Pearson $r$ is listed first followed by the Spearman rho.

884 Correlations $\geq .7$ are acceptable and below 0.3 as unacceptable. RT: Response Time; TOAV:

885 Test of Attentional Vigilance; Trail Making Test Ratio of Completion times for Part B/Part A $886(\mathrm{~B} / \mathrm{A})$.

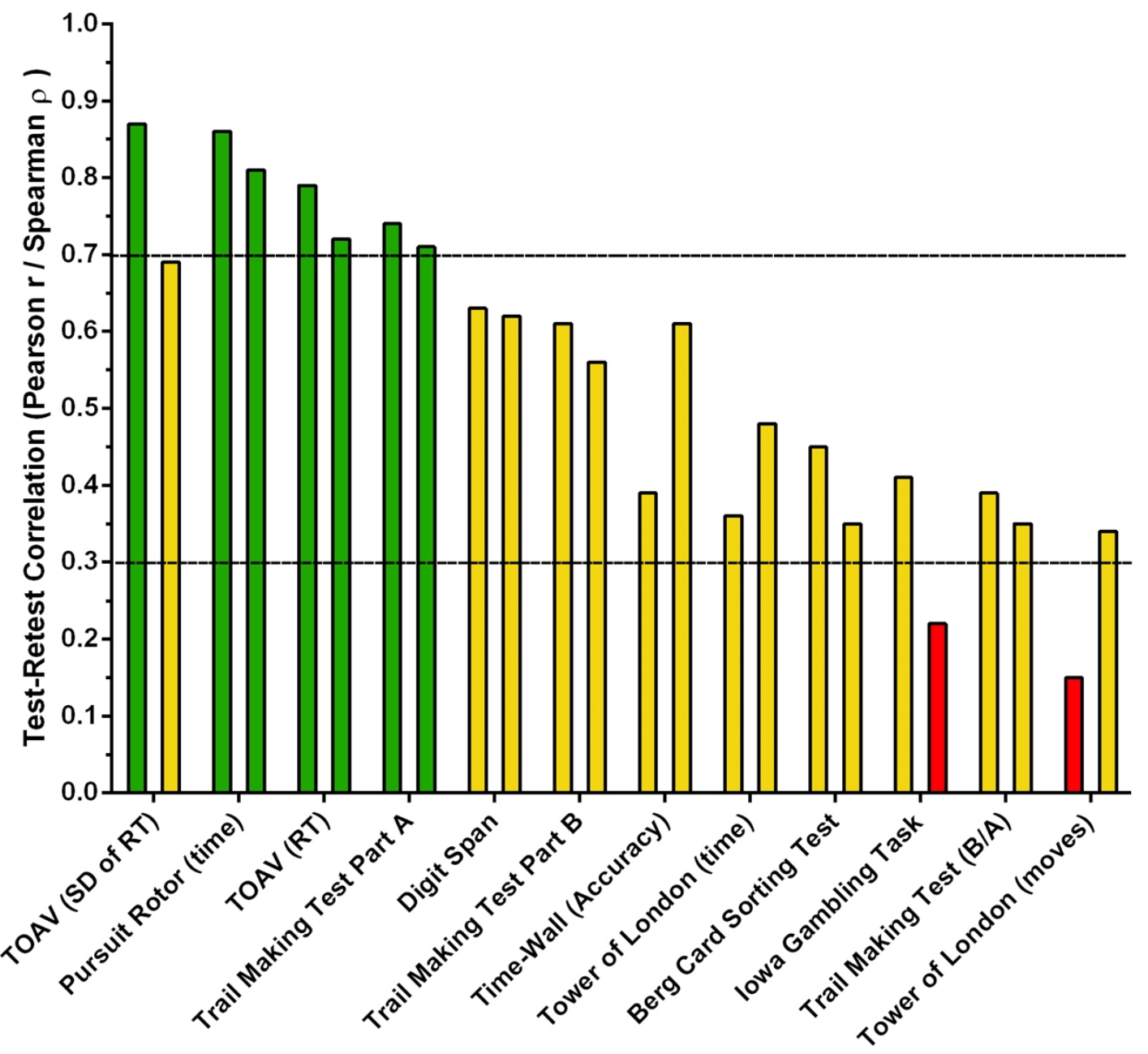


888 Figure 4. Change from the test to the retest, expressed as Cohen's $d$ measure of effect size,

889 among young-adults completing the Psychology Experiment Building Language (PEBL)

890 neurobehavioral test battery. Paired t-test ${ }^{\mathrm{A}} P<.05,{ }^{\mathrm{B}} P<.01$.

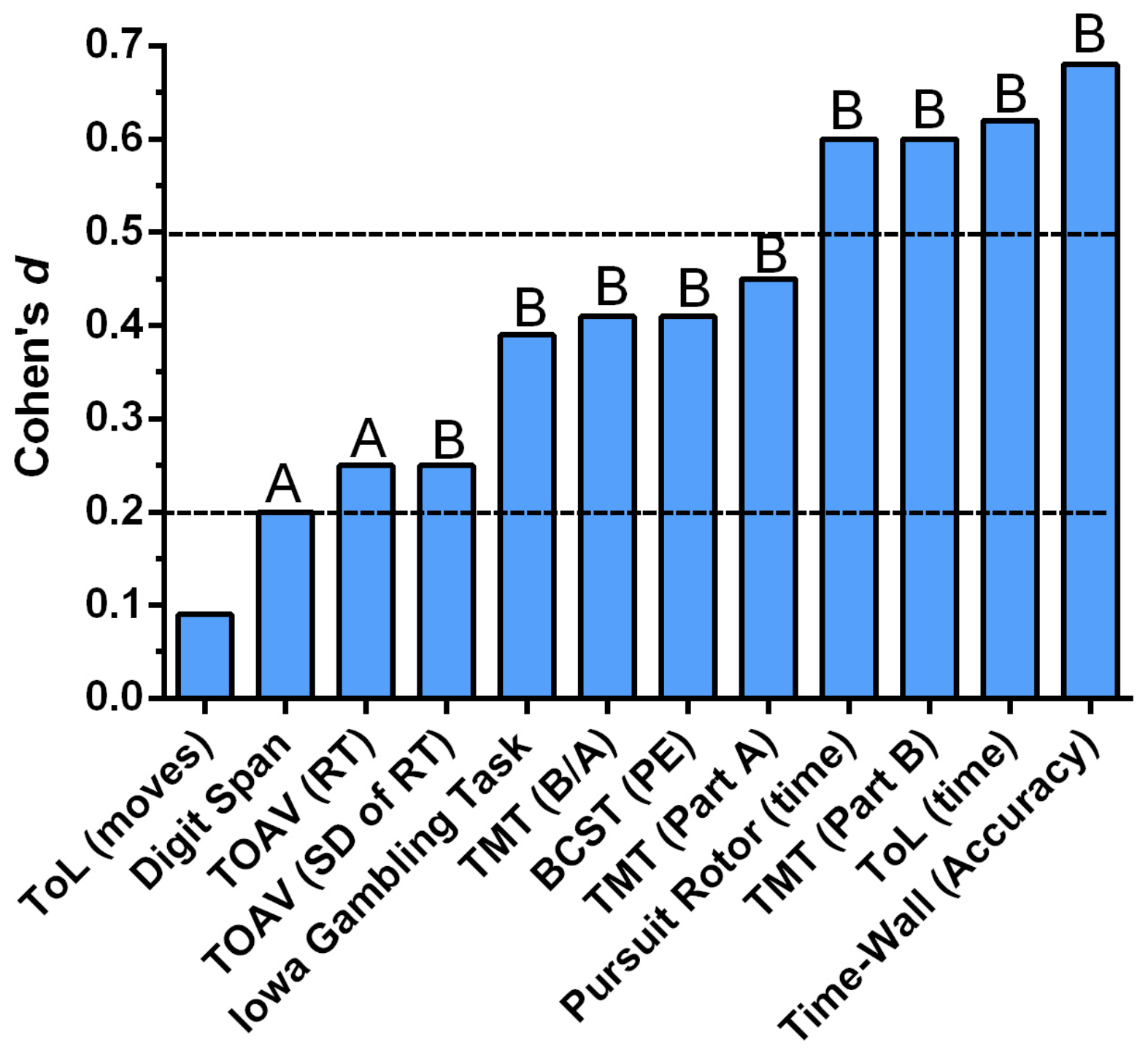

891 
